\title{
Rehabilitation of Patients with Geriatric Foot Syndrome in Out-patient
} Settings

\author{
${ }^{1}$ Irina P. Pochitaeva, ${ }^{2}$ Andrei N. Ilnitskii, ${ }^{*}$ Svetlana G. Gorelik, ${ }^{4}$ Margarita V. Koroleva, ${ }^{5}$ Elmira E. \\ Satardinova, ${ }^{6}$ Elena A. Voronina \\ ${ }^{1,2,4}$ Academy of Postgraduate Education of the Federal State Budgetary Institution "Federal Scientific and Clinical \\ Center for Specialized Types of Medical Care and Medical Technologies of the Federal Biomedical Agency", \\ Volokolamskoe Highway 91, Moscow, 125371 \\ ${ }^{3}$ Federal State Autonomous Educational Institution of Higher Education "Belgorod State National Research \\ University” (NRI BelSU), Pobedy Str. 85, Belgorod, 308015, Russia \\ ${ }^{5}$ Irkutsk State Medical Academy of Postgraduate Education, Russia, 664049, Irkutsk, Yubileiny Microdistrict, 100 \\ ${ }^{6}$ Department of Social Protection of the Population of Kemerovo Region, Kuznetsky Ave. 19 A, Kemerovo, 650991 \\ Email: 1info@belsu.ru, ${ }^{2}$ depart@dsznko.ru, ${ }^{3}$ sggorelik@mail.ru, ${ }^{4}$ irkmapo@irk.ru, \\ russia@prescopus.com, ${ }^{5}$ info@medprofedu.ru
}

Received: 14th November 2019, Accepted: 04th December 2019, Published: 31st December 2019

\begin{abstract}
Geriatric (senile) foot, according to various authors, is one of the most common syndromes in the elderly and senile. This geriatric syndrome is polyetiological since local factors take part in its formation - circulatory disorders in the distal extremities, osteoarthritis of the small joints of the foot, age-related decrease in skin sensitivity and other, as well as somatic diseases leading to the development of geriatric syndromes such as dizziness, ataxia, imbalance, which contributes to repeated falls. This article describes the developed and scientifically substantiated tactics of rehabilitation treatment of patients with age-related foot syndrome in a clinic, which consists in identifying geriatric syndromes associated with age-related foot syndrome and the use of a set of measures to prevent their progression and treatment. The following recommendations were added to targeted secondary prevention: 1) technical means of rehabilitation; 2) orthopedic shoes; 3) morning exercises and passive movements in the limbs; 4) the use of canes, walkers. All these measures are recommended for the prevention of the syndrome of falls and sarcopenia, the correction of walking disorders and an increase in general motor activity. Nutritional support, nutrition status correction, and nutrition monitoring are essential for the prevention and correction of malnutrition syndrome. Together, the above additional measures had a clear focus on the prevention and correction of senile asthenia syndrome.
\end{abstract}

\section{Keywords}

Geriatric Foot, Senile Asthenia, Geriatric Syndromes, Rehabilitation, Old Age, Senile Age.

\section{Introduction}

Modern approaches to the rehabilitation treatment of elderly and senile people develop along with providing syndromic care, which is ultimately focused on maintenance of the maximum degree of independence and shaping of the highest possible quality of life [1,2].

Geriatric (senile) foot, according to various authors, is one of the most common syndromes in the elderly and senile. This geriatric syndrome is polyetiological since local factors take part in its formation - circulatory disorders in the distal extremities, osteoarthritis of the small joints of the foot, age-related decrease in skin sensitivity and other, as well as somatic diseases leading to the development of geriatric syndromes such as dizziness, ataxia, imbalance, which contributes to repeated falls $[3,4]$.

In addition, the domestic literature does not almost distinguish geriatric foot syndrome as an object of rehabilitation treatment. For the first time in domestic literature, geriatric foot syndrome was described in 2014 [5]. To date, there are only a few works that highlight aspects of rehabilitation treatment in this condition. In addition, these programs neglect the prevention of precisely those geriatric syndromes, which then lead to the formation of senile asthenia syndrome, and the development of this condition determines the degree of social insufficiency in elderly and senile people. On the other hand, rehabilitation treatment has the fullest potential for the rehabilitation of the functionality of people of older age groups, which is reflected in the World Health Organization Global Report on Aging (2015) $[6,7]$.

Based on the foregoing, the development and justification of comprehensive rehabilitation treatment programs for geriatric foot syndrome aimed at preventing the development of senile asthenia syndrome and geriatric syndromes leading to this condition are timely and relevant.

The objective of our study is to develop and scientifically substantiate the tactics of rehabilitation treatment of patients with geriatric foot syndrome in a clinic. 


\section{Material and Methods}

This study consisted of three sequential steps.

The first stage is to study age-related features of the development of geriatric foot syndrome. The study at this stage was prospective in nature, included five groups of patients using a step-by-step age-related analysis with a 5-year interval, aged 55 to 79 years, the average age was $68.6 \pm 6.5$ years, the sample size was 298 people: 145 men and 153 women. The following was studied in patients: common complaints (intermittent claudication, pain in the feet or foot joints at rest or during movement, trophic disorders, swelling of the legs and feet); the appearance of the foot (assessment of the degree of muscle hypotrophy, subcutaneous fat, detection of skin changes (hypotrichosis, hyperkeratosis); objective signs of damage to the nail plates, determination of pulsation on the main arteries at standard points, the presence of signs of venous pathology; special functional tests (Oppel); laboratory examination (determination of the serum lipid profile, glucose level, highly sensitive C-reactive protein); instrumental examination (rheovasography of lower extremities, Doppler ultrasound and Dopplerometry); examination by specialist doctors to identify concomitant pathology (cardiologist, endocrinologist, neurologist); specialized geriatric examination (identification of age-related conditions, such as fall syndrome, malnutrition, sarcopenia, anxiety-depressive syndrome, senile asthenia syndrome) [8].

Identification of senile asthenia syndrome and other geriatric syndromes was carried out by examining and interviewing elderly and senile patients using the original computer program - "Optimization of care in geriatrics depending on the degree of senile asthenia" based on specialized geriatric examination (state registration certificate No. 2013660311).

This stage of the study ended with the identification of age-specific features of the development of clinical manifestations of geriatric foot syndrome in the selected groups based on an integrated approach.

At the second stage of the study, we conducted a statistical factor analysis of the generated sample (298 patients), which allowed us to identify the main risk factors for impaired function in patients with age-related foot syndrome, depending on the age of the patients. Also, on the basis of a retrospective study of the current medical outpatient documentation (medical record of an outpatient patient F. 025/y), existing approaches to the correction of patients with geriatric foot syndrome were investigated. Based on expert assessment, new comprehensive principles were recommended for optimizing the management of elderly and senile patients with geriatric foot syndrome requiring rehabilitation treatment.

At the third stage, we developed and optimized management of patients with geriatric foot syndrome, its implementation and effectiveness assessment. To do this, a prospective, randomized, controlled study was conducted. The patients included were between the ages of 70 and 79 , the average age was $75.6+2.3$ years, the sample population was 72 patients. Inclusion criteria: the presence of a diagnosed geriatric foot syndrome with a leading vascular component (against the background of obliterating atherosclerosis of the vessels of the lower extremities of the initial stages with chronic arterial insufficiency of the I-II stage), the presence of mild asthenia syndrome.

Exclusion criteria: age-related foot syndrome with a leading neurological or venous component, the presence of severe obliterating atherosclerosis of lower limb vessels with chronic arterial insufficiency above stage II, the presence of moderate and severe senile asthenia syndrome or absence of senile asthenia syndrome, severe general somatic status, severe cognitive deficiency hampering contact with the patient.

The medical and social characteristics of the patients included in this section of the study are presented in Table 1 .

\begin{tabular}{|l|c|c|c|c|}
\hline \multirow{2}{*}{$\begin{array}{c}\text { Medical and social } \\
\text { parameter }\end{array}$} & \multicolumn{2}{c|}{ Control group (n=35) } & \multicolumn{2}{c|}{ Main group (n=37) } \\
\cline { 2 - 5 } & Abs. & $\%$ & Abs. & \multicolumn{2}{c|}{$75.1 \pm 2.1$} \\
\hline Age & \multicolumn{2}{|c|}{$75.9 \pm 1.8$} & 12 & 32.4 \\
\hline Men & 11 & 31.4 & 25 & 67.6 \\
\hline Women & 24 & 68.6 & 27 & 72.9 \\
\hline Disable & 23 & 65.7 & 18 & 48.6 \\
\hline Living alone & 19 & 54.3 & 21 & 56.8 \\
\hline Concomitant arterial hypertension & 20 & 57.1 & 17 & 45.9 \\
\hline Concomitant ischemic heart disease & 18 & 51.4 & 10 & 27.0 \\
\hline Concomitant obesity & 11 & 31.4 & & \\
\hline
\end{tabular}

Table 1: Medical and Social Characteristics of Patients (abs., \%)

Patients of the main group $(n=37)$ with geriatric foot syndrome underwent rehabilitation treatment optimized by us, which consisted of the following: a) a comprehensive assessment of the patient's status upon rehabilitation treatment, including a specialized geriatric examination; b) diet therapy; c) jogging; g) 10 sessions of magnet-laser therapy; e) optimization of drug therapy (prescribing statins (atorvastatin $10 \mathrm{mg}$ or rosuvastatin $5 \mathrm{mg}$ ), optimizing hypoglycemic therapy (achieving target figures for glycosylated hemoglobin), prescribing analgesics for effective relief of pain, thorough medical correction of general somatic status, treatment of osteoporosis) d) identification of geriatric syndromes associated with geriatric foot syndrome, primarily syndromes of fall, malnutrition and sarcopenia, anxiety- 
depressive syndrome, senile asthenia syndrome, and the use of a set of measures to prevent their progression (technical rehabilitation equipment, orthopedic shoes, monitoring nutrition, nutritional support, morning exercises, prescription of modern antidepressants (serotonin-fluoxetine reuptake inhibitors $20 \mathrm{mg} / \mathrm{day}$, ALSI Farma, Russia). The course of treatment was 5 months.

Patients in the control group $(n=35)$ were not referred to rehabilitation treatment units and received treatment for identified diseases as part of standard medical observation programs. The course of treatment was also 5 months.

Efficiency assessment in the main and control groups was carried out by comparing the indicators before the study and after 5 months. In terms of the effectiveness of therapy and measures, patients were examined according to the "Lower limb functional scale" [5] and evaluated according to changes in geriatric status and the development of geriatric syndromes such as syndromes of fall, malnutrition, sarcopenia, anxiety-depressive syndrome. In addition to the comparative assessment of functional changes in geriatric foot syndrome and geriatric status, an assessment of the quality of life was carried out using the SF 36 scale.

Statistical processing of the research results included the calculation of average absolute and relative values with the calculation of the average error; determination of the nature of distribution using the chi-square method; assessment of the significance of differences between the two sets according to the Student t-test; and factor analysis with the calculation of the F coefficient.

\section{Results and Discussion}

Typical complaints of elderly patients are presented in Table 2.

According to the data obtained, the subjective symptoms of pathological processes in the foot area increased with aging, reaching their maximum at the age of 75-79 years.

An objective assessment of local status (status localis), performed by the author, using a visual analogue scale, are presented in Table 3.

The results obtained indicate a correlation of objective data with patient complaints and an increase in local pathological phenomena as they age. This is also confirmed by the Oppel functional test (a symptom of plantar ischemia), indicating a significant contribution to the development of geriatric foot syndrome of the vascular component in connection with the formation of chronic arterial insufficiency against the background of obliterating atherosclerotic process (Fig. 1).

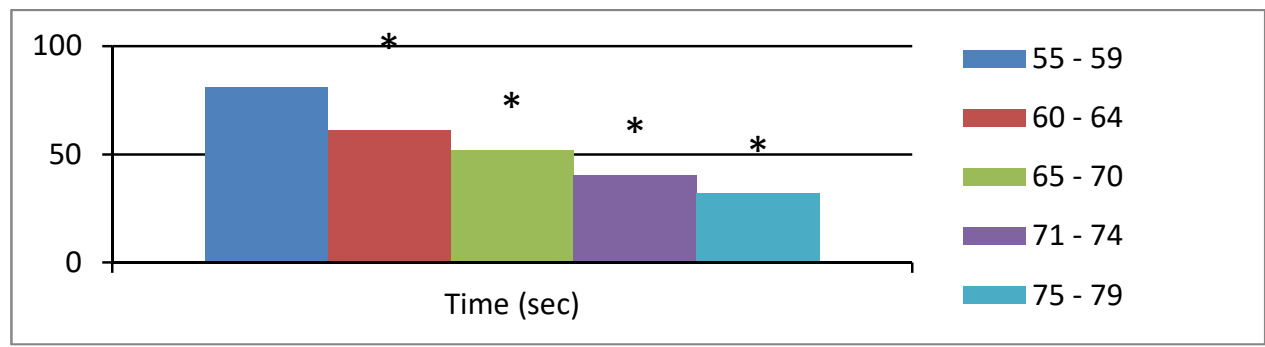

${ }^{*} \mathrm{p}<0.05$ when comparing the indicator in a stepwise age analysis in the studied age group compared with the previous age group

Figure 1: Age-related Dynamics of a Symptom of Plantar Ischemia (Seconds).

\begin{tabular}{|l|c|c|c|c|c|}
\hline \multirow{2}{*}{ Patients' complaints } & \multicolumn{5}{|c|}{ Age period (years) } \\
\cline { 2 - 6 } & $55-59$ & $60-64$ & $65-69$ & $70-74$ & $75-79$ \\
\hline $\begin{array}{l}\text { Intermittent } \\
\text { claudication (m) }\end{array}$ & $\begin{array}{c}1241.1 \\
\pm 21.4\end{array}$ & $\begin{array}{c}1012.2 \\
\pm 10.7^{*}\end{array}$ & $\begin{array}{c}901.4 \\
\pm 6.1^{*}\end{array}$ & $802.9 \pm 6.4^{*}$ & $625.8 \pm 7.3^{*}$ \\
\hline $\begin{array}{l}\text { Pain in the joints } \\
\text { (points) }\end{array}$ & $\begin{array}{c}1.2 \pm \\
0.1\end{array}$ & $1.9 \pm 0.2^{*}$ & $2.2 \pm 0.1^{*}$ & $3.1 \pm 0.2^{*}$ & $4.0 \pm 0,3^{*}$ \\
\hline $\begin{array}{l}\text { Trophic disorders } \\
\text { (points) }\end{array}$ & $1.3 \pm 0,2$ & $1.8 \pm 0.3^{*}$ & $2.5 \pm 0.2^{*}$ & $3.2 \pm 0.1^{*}$ & $4.1 \pm 0.2^{*}$ \\
\hline $\begin{array}{l}\text { Edematous syndrome } \\
\text { (points) }\end{array}$ & $1.1 \pm 0.2$ & $1.7 \pm 0.3^{*}$ & $2.4 \pm 0.2^{*}$ & $3.1 \pm 0.2^{*}$ & $4.2 \pm 0.1^{*}$ \\
\hline
\end{tabular}

$* \mathrm{p}<0.05$ when comparing the indicator in a stepwise age analysis in the studied age group compared with the previous age group

Table 2: Age-related Analysis of Patient's Complaints during the Formation of Geriatric Foot Syndrome (Points, $\mathbf{M} \pm \mathbf{m}$ ) 


\begin{tabular}{|l|c|c|c|c|c|}
\hline \multirow{2}{*}{ Parameter } & \multicolumn{5}{|c|}{ Age period (years) } \\
\cline { 2 - 6 } & $55-59$ & $60-64$ & $65-69$ & $70-74$ & $75-79$ \\
\hline Hypotrophy (points) & $1.2 \pm 0.3$ & $1.8 \pm 0.2^{*}$ & $2.5 \pm 0.1^{*}$ & $3.2 \pm 0.2^{*}$ & $4.0 \pm 0.1^{*}$ \\
\hline Skin changes (points) & $1.1 \pm 0.2$ & $1.7 \pm 0.1^{*}$ & $2.6 \pm 0.2^{*}$ & $3.3 \pm 0.1^{*}$ & $4.1^{*} 0.2^{*}$ \\
\hline Nail changes (points) & $1.2 \pm 0.2$ & $1.8 \pm 0.1^{*}$ & $2.6 \pm 0.3^{*}$ & $3.2 \pm 0.1^{*}$ & $4.2 \pm 0.1^{*}$ \\
\hline $\begin{array}{l}\text { Venous pathology } \\
\text { (points) }\end{array}$ & $1.7 \pm 0.1$ & $2.3 \pm 0.2^{*}$ & $2.9 \pm 0.1^{*}$ & $3.5 \pm 0.1^{*}$ & $4.5 \pm 0.2^{*}$ \\
\hline $\begin{array}{l}\text { Decreased heart rate (\% } \\
\text { of normal) }\end{array}$ & $74.1 \pm 2.5$ & $61.5 \pm 2.3^{*}$ & $50.4 \pm 2.1^{*}$ & $40.5 \pm 2.2^{*}$ & $31.4 \pm 2.1^{*}$ \\
\hline
\end{tabular}

${ }^{*} \mathrm{p}<0.05$ when comparing the indicator in a stepwise age analysis in the studied age group compared with the previous age group

Table 3: Age-related Analysis of Local Status during the Formation of Geriatric Foot Syndrome (Points, $\mathbf{M} \pm \mathbf{m})$

The results of the instrumental examination - rheovasography of the vessels of the lower extremities, ultrasound dopplerography, and heel bone densitometry indicate significantly more pronounced changes in the vascular system of the foot and the state of bone tissue at the age of 70-79 years. In particular, according to the results of rheovasography, patients aged 55-69 years had a compensated type of change in arterial and venous blood flow, and older age groups had its subcompensation (Table 4).

\begin{tabular}{|l|l|l|l|l|l|}
\hline \multirow{2}{*}{\multicolumn{1}{c|}{$\begin{array}{c}\text { Blood flow } \\
\text { parameters }\end{array}$}} & \multicolumn{5}{|c|}{ Age period (years) } \\
\cline { 2 - 6 } & $55-59$ & $60-64$ & $65-69$ & $70-74$ & $75-79$ \\
\hline Rheographic index & $0.04 \pm 0.01$ & $0.05 \pm 0.02$ & $0.04 \pm 0.02$ & $0.03 \pm 0.02^{*}$ & $0.03 \pm 0.02^{*}$ \\
\hline Elasticity index & $0.2 \pm 0.02$ & $0.2 \pm 0.03$ & $0.2 \pm 0.03$ & $0.1 \pm 0.01^{*}$ & $0.1 \pm 0.02 *$ \\
\hline Venous outflow index & $0.3 \pm 0.02$ & $0.4 \pm 0.03$ & $0.3 \pm 0.02$ & $0.6 \pm 0.01 *$ & $0.6 \pm 0.02 *$ \\
\hline
\end{tabular}

${ }^{*} \mathrm{p}<0.05$ when comparing the indicator in a stepwise age analysis in the studied age group compared with the previous age group

Table 4: Age-related Dynamics of the State of Regional Blood Flow in the Feet $(M \pm m$, Points)

The laboratory examination revealed the presence of atherogenic dyslipoproteinemia in all age groups. In particular, the average total cholesterol values were $7.2 \pm 0.6 \mathrm{mmol} / \mathrm{L}$, the average $\mathrm{LDL}$ values were $4.2 \pm 0.4 \mathrm{mmol} / \mathrm{L}$, and the average HDL values were $0.8 \pm 0.2 \mathrm{mmol} / \mathrm{L}$. The results obtained testified to the contribution of atherogenic dyslipoproteinemia to the genesis of geriatric foot syndrome. We emphasize that there were no statistically significant age-related differences in the values of these indicators between the studied age groups.

The results correlated with heel bone densitometry data according to the standard deviation (SD) according to the Student t-test. The age group of 55-59 years old had this indicator equal to $0.8 \pm 0.1$ (normal), $60-64$ years old $-1.3 \pm 0.2$ (osteopenia), 65-69 years old - $1.5 \pm 0.1$ (osteopenia), 70-74 years old - $1.8 \pm 0.2$ (osteopenia), $75-79$ years old - $2.6 \pm 0.4$ (moderate osteoporosis), $\mathrm{p}<0.05$ as age increases.

We revealed that, with an increase in age, concomitant diseases developed too, which ultimately led to the formation of common geriatric syndromes. Most common nosological forms were coronary heart disease, hypertension, type II diabetes, thyroid hyperplasia, dorsopathy, and their prevalence increased along with age (Table 5).

\begin{tabular}{|l|c|c|c|c|c|}
\hline \multicolumn{1}{|c|}{ Pathology } & \multicolumn{5}{|c|}{ Age period (years) } \\
\cline { 2 - 6 } & $55-59$ & $60-64$ & $65-69$ & $70-74$ & $75-79$ \\
\hline $\begin{array}{l}\text { Ischemic heart } \\
\text { disease }\end{array}$ & $25.7 \pm 2.4$ & $27.1 \pm 2.5^{*}$ & $35.4 \pm 2.7^{*}$ & $41.4 \pm 1.5^{*}$ & $57.2 \pm 2.1^{*}$ \\
\hline $\begin{array}{l}\text { Arterial } \\
\text { hypertension }\end{array}$ & $41.3 \pm 2.7$ & $42.4 \pm 2.9^{*}$ & $50.1 \pm 3.1^{*}$ & $60.2 \pm 3.2^{*}$ & $71.4 \pm 3.1^{*}$ \\
\hline $\begin{array}{l}\text { Type II diabetes } \\
\text { mellitus }\end{array}$ & $21.2 \pm 1.5$ & $22.5 \pm 1.4^{*}$ & $32.4 \pm 1.5^{*}$ & $39.5 \pm 1.4^{*}$ & $51.1 \pm 1.5^{*}$ \\
\hline $\begin{array}{l}\text { Thyroid gland } \\
\text { hyperplasia }\end{array}$ & $20.8 \pm 1.6$ & $21.4 \pm 1.7^{*}$ & $30.1 \pm 1.4^{*}$ & $37.8 \pm 1.3^{*}$ & $49.7 \pm 1.4^{*}$ \\
\hline Dorsopathy & $35.4 \pm 1.5$ & $36.2 \pm 1.6^{*}$ & $42.4 \pm 1.3^{*}$ & $50.2 \pm 1.1^{*}$ & $61.4 \pm 1.6^{*}$ \\
\hline
\end{tabular}

$*_{\mathrm{p}}<0.05$ when comparing the indicator in a stepwise age analysis in the studied age group compared with the previous age group

Table 5: Age-related Dynamics of the Prevalence of Concomitant Diseases in the Formation of Geriatric Foot Syndrome (\%, M \pm m) 
Specialized geriatric examination revealed that the number of general geriatric syndromes increases along with aging and reaches its peak in age groups of 70 years and older. The most relevant concomitant common geriatric syndromes in the geriatric foot syndrome were syndrome of fall, malnutrition syndrome, anxiety-depressive syndrome, and senile asthenia syndrome (Table 6).

\begin{tabular}{|l|c|c|c|c|c|}
\hline \multicolumn{1}{|c|}{$\begin{array}{c}\text { General geriatric } \\
\text { syndrome }\end{array}$} & \multicolumn{5}{|c|}{ Age period (years) } \\
\cline { 2 - 6 } & $55-59$ & $60-64$ & $65-69$ & $70-74$ & $75-79$ \\
\hline Syndrome of fall & $2.1 \pm 0.1$ & $4.1 \pm 0.2^{*}$ & $6.1 \pm 0.3^{*}$ & $15.8 \pm 1.4^{*}$ & $28.1 \pm 2.5^{*}$ \\
\hline Malnutrition syndrome & $3.4 \pm 0.2$ & $6.8 \pm 0.9^{*}$ & $12.1 \pm 1.5^{*}$ & $21.9 \pm 1.7^{*}$ & $39.1 \pm 1.5^{*}$ \\
\hline $\begin{array}{l}\text { Anxiety depressive } \\
\text { syndrome }\end{array}$ & $20.1 \pm 0.3$ & $4.0 \pm 0.3^{*}$ & $6.3 \pm 0.4^{*}$ & $17.1 \pm 1.5^{*}$ & $31.5 \pm 2.1^{*}$ \\
\hline Sarcopenia syndrome & $0.5 \pm 0.02$ & $2.1 \pm 0.5^{*}$ & $4.1 \pm 1.2^{*}$ & $7.3 \pm 1.4^{*}$ & $10.5 \pm 1.2^{*}$ \\
\hline $\begin{array}{l}\text { Senile asthenia } \\
\text { syndrome }\end{array}$ & - & $2.2 \pm 0.7^{*}$ & $4.5 \pm 1.1^{*}$ & $18.9 \pm 1.5^{*}$ & $32.5 \pm 1.1^{*}$ \\
\hline
\end{tabular}

${ }^{*} \mathrm{p}<0.05$ when comparing the indicator in a stepwise age analysis in the studied age group compared with the previous age group

Table 6: Age-related Dynamics of the Prevalence of Common Geriatric Syndromes (\%, M \pm m)

We found that as the age increases, the number of patients with the pathologies of legs and feet increases too, which is confirmed by the data of objective examination and the results of laboratory and instrumental tests. At the age of 70-79 years, there was also a significant increase in the incidence of common geriatric syndromes. The data obtained allow us to conclude that with aging, the formation of the syndrome of the age foot occurs, which leads to a deterioration in the general geriatric status.

Based on the data obtained, we optimized the rehabilitation treatment with medical and social support for patients aged 70-79 with geriatric foot syndrome, which consisted in identifying geriatric syndromes associated with geriatric foot syndrome, primarily syndromes of fall, malnutrition and sarcopenia, anxiety-depressive syndrome, senile asthenia syndrome, in the application of a set of measures aimed at their prevention and treatment. The following recommendations were added to targeted secondary prevention in patients aged 55-69 years: 1) technical means of rehabilitation; 2) orthopedic shoes; 3) morning exercises and passive movements in the limbs; 4) the use of canes, walkers. All these measures are recommended for the prevention of the syndrome of falls and sarcopenia, the correction of walking disorders and an increase in general motor activity. Nutritional support, nutrition status correction, and nutrition monitoring essential for the prevention and correction of malnutrition syndrome were also applied.

Together, the above additional measures had a clear focus on the prevention and correction of senile asthenia syndrome. Medication approaches included the prescription of modern selective serotonin reuptake inhibitors in the presence of anxiety-depressive syndrome (fluoxetine $20 \mathrm{mg}$ /day, CJSC ALSI Farma, Russia).

\section{Conclusion}

The effectiveness of the proposed optimized rehabilitation treatment of patients with geriatric foot syndrome receiving medical and social support, which consists in the correction of non-drug and drug therapy, was tested by us in the age group of 70-79 years as a target in the provision of specialized geriatric care. In terms of the effectiveness of therapy and measures, patients were examined according to the "Lower limb functional scale" [5] and evaluated according to changes in geriatric status and the development of geriatric syndromes such as syndromes of fall, malnutrition, sarcopenia, anxiety-depressive syndrome, and senile asthenia syndrome. In addition to the comparative assessment of functional changes in geriatric foot syndrome and geriatric status, an assessment of the quality of life was carried out using the SF 36 scale.

It was found that the "Functional scale for the lower limb" in the control group estimated difficulties associated with the state of the lower limb (housekeeping, using the bathroom and toilet, walking, standing on legs, going up and down stairs, putting on socks, etc.) before the start of the observation as $42.5 \pm 2.3$ points, after 5 months $-41.7 \pm 2.2$ points, in the main group - $42.6 \pm 2.7$ points and $61.4 \pm 2.4$ points, respectively, $\mathrm{p}<0.05$.

The assessment of geriatric status over time during the optimized rehabilitation treatment of patients with geriatric foot syndrome receiving medical and social support found that in the control group the incidence of the syndrome of fall before and after the observation was $32.8 \pm 2.4 \%$ and $33.4 \pm 3.1 \%$, respectively, malnutrition syndrome $-35.0 \pm 2.7 \%$ and $33.8 \pm 2.6 \%$, anxiety-depressive syndrome $-32.1 \pm 2.1 \%$ and $31.9 \pm 2.0 \%$; in the main group, the incidence of syndrome of fall was $32.7 \pm 2.3 \%$ and $20.4 \pm 2.1 \%$, malnutrition syndrome $-34.5 \pm 2.6 \%$ and $19.1 \pm 2.4 \%$, anxietydepressive syndrome $-31.8 \pm 2.0 \%$ and $18.5 \pm 2.3 \%, \mathrm{p}<0.05$. The study also revealed that the severity of sarcopenic syndrome and senile asthenia after 5 months of observation in the control group did not change, while in the main group it decreased by $12.5+1.2 \%$ and $10.5+1.0 \%$, respectively, $<0.05$.

We also obtained data according to which the introduction of an optimized rehabilitation treatment strategy for patients with geriatric foot syndrome leads to a significant improvement in the quality of life. Comparison of the indicators in the control group detected no significant changes. In the main group, comparison of the indicators before and after the 
optimized treatment revealed a significant improvement in physical functioning $(39.9 \pm 8.9$ and $68.2 \pm 23.1$, respectively), role-based functioning due to physical condition ( $52.8 \pm 10.9$ and $72.8 \pm 27.5$, respectively), pain severity $(63.5 \pm 18.5$ and $85.1 \pm 29.3$, respectively) and general health status (45.6 \pm 10.1 and $68.2 \pm 23.1$, respectively), $\mathrm{p}<0.05$. All these indicators showed a significant improvement in the physical component of health. A significant improvement was also in vital activity (34.6 \pm 6.9 and $71.7 \pm 26.7$, respectively), social functioning (36.0 \pm 7.4 and $69.9 \pm 24.8$, respectively), role-based functioning due to the emotional state (47.0 \pm 10.7 and $72.2 \pm 27.1$, respectively), and mental health $(61.8 \pm 19.6$ and $74.6 \pm 28.8$, respectively), $\mathrm{p}<0.05$. All these indicators constituted a significant improvement in the psychological component of health.

Thus, provision of medical care in the clinical settings to elderly and senile patients with geriatric foot syndrome should consider different approaches to the management of certain age categories of such patients. The developed and tested optimized rehabilitation treatment for patients with age-related foot syndrome, which under the age of 70 years consists in identifying risk factors for its development and conducting targeted secondary prevention (vascular, bone, neurological, biochemical), and after 70 years - in identifying the risks of progression of geriatric foot syndrome in the context of general geriatric status with the subsequent use of optimized rehabilitation treatment tactics with medical and social support, which consists in the correction of non-drug and drug therapy, leads to an improvement in the functionality of the lower limb, a decrease in the frequency of general geriatric syndromes and helps to improve the quality of life of patients.

\section{References}

1. Shabalin V.N. Organization of the work of the geriatric service in progressive demographic aging of the population of the Russian Federation. Successes in gerontology. 2009. V. 22, No. 1. 186-195 pp.

2. A guidance to gerontology and geriatrics: in 4 volumes; under ed. Acad. RAMS, Prof. V.N. Iarygin, Prof. A.S. Melentiev. M.: GEOTAR-Media, 2008. V. 4. Clinical Geriatrics. 528 p.

3. Ilnitskii, A.N. Specialized Geriatric Examination. V.F. Kuprevich Gerontological Journal. 2012. No. 4-5. 66-84 pp. 4. Redkin Iu.A. Diabetic foot syndrome: diagnosis, treatment, prevention. Quality of life. Medicine. 2003. No. 1. 33$41 \mathrm{pp}$.

5. Proshchaev K., et al. Geriatric foot syndrome in aesthetic medicine. Aesthetic Medicine, 2014. N 1. 61-65 pp.

6. On approval of the Procedure for the provision of medical care in the geriatrics profile: Order of the Ministry of Health of Russia No. 38H dated 01.29.2016 (Registered in the Ministry of Justice of Russia on March 14, 2016 under No. 41405).

7. The 2025 Action Strategy in the interests of senior citizens in the Russian Federation dated February 5, 2016 , No. 164-p. ConsultantPlus: Internet version: legal reference system [Electronic resource]. - Access mode: http://www.consultant.ru/document/cons_doc_LAW_193464/

8. Geriatric syndromes in older surgical patients: a literature review / P. J. Mcrae, A. M. Mudge, N. M. Peel [et al]. J. of Frailty and Aging. 2013. Vol 2, № 4. 205-210 pp. 